\title{
A HIGH TUNING-RATIO SILICON-MICROMACHINED VARIABLE CAPACITOR WITH LOW DRIVING VOLTAGE
}

\author{
Zhihong Li, and Norman C. Tien \\ Berkeley Sensor \& Actuator Center and Department of Electrical and Computer Engineering, \\ University of California, Davis, CA 95616.
}

\begin{abstract}
A bulk-micromachined variable capacitor, in which the gap distance between parallel plates is adjusted by comb-drive actuators, has been developed. This capacitor possesses the merits of parallel-plate capacitors and comb-drive actuation without their demerits. Therefore, this device has high tuning range and low tuning voltages with a small footprint and stiff springs, resulting in lower mechanical noise sensitivity and high tuning speed. The initial fabricated devices demonstrate a 5:1 tuning ratio at $20 \mathrm{~V}$ with $8.46 \mathrm{pF}$ maximum capacitance.
\end{abstract}

\section{INTRODUCTION}

Variable capacitors have wide applications in RF circuits for wireless communications such as low-noise voltage-controlled oscillators (VCO's) and high dynamic range filters. Micromachined variable capacitors are very attractive due to their advantages of low parasitics, large voltage swing and high $Q$ [1-8]. The main objectives for a micromachined variable capacitor are to achieve low driving voltage, high tuning ratio, and low mechanical noise sensitivity.

Micromachined variable capacitors generally fall in two categories - those that vary the area and those that vary gap [2, 3]. Often, gaptuning capacitors are constructed from two surface-micromachined electrostatically-actuated parallel plates. One drawback of this method is that the movable plate can only be actuated one-third of the initial gap distance; beyond this value, the two plates will snap together. In this case, the maximum theoretical tuning ratio is only 1.5:1. In practice, the tuning range can be even lower because of electrical overshoot or mechanical shock. Although techniques that increase the tuning range have been reported $[4,5]$, they often do not have tuning ratios large enough for many RF applications, and furthermore, have increased process complexity.

Most area-tuning capacitors utilize comb drives as the actuation component and another set of comb fingers as the capacitor [3, 8]. This type of capacitor has no theoretical limitation on the tuning range, because the driving force of comb drives is independent of displacement $[9,10]$. However, the capacitance per unit area of comb fingers is typically smaller than parallel plates because of larger gap distances. In addition, the actuation voltage is larger for the same tuning ratio because larger displacement is required. A larger capacitance can be obtained by increasing the device area, while the driving voltage can be lowered by increasing the device area and decreasing the stiffness of springs. However, the larger mass and lower stiffness will lead to higher mechanical noise sensitivity and lower tuning speed because of the decreased resonant frequency.

In this paper, we present a bulk-micromachined variable capacitor that employs comb drive actuators to laterally displace parallel plate capacitors. This device is realized via a simple singlecrystal-silicon process performed on glass substrate. This process allows the fabrication of high performance devices on a low loss substrate.

\section{DEVICE DESIGN}

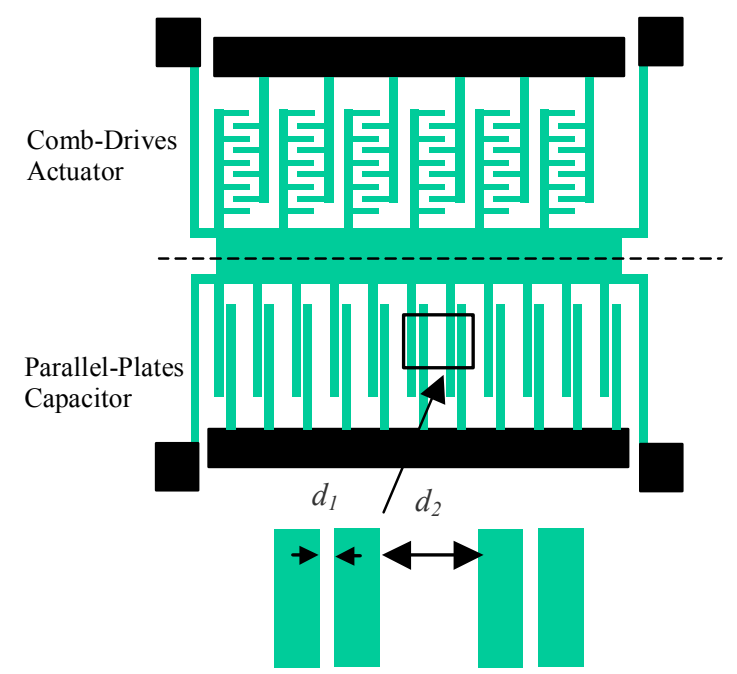

Fig. 1 The schematic view of the variable capacitor with parallel plates as capacitors and comb drives providing the tuning.

Fig. 1 shows a schematic view of our designed capacitor. In this capacitor, comb drives provide actuation by the well-known equation $[9,10]$

$$
F=\frac{\partial C}{\partial x} \frac{V^{2}}{2}=n \frac{h \varepsilon_{0}}{g} V^{2}
$$

where $n$ is the number of the fingers in the tuning structure, $g$ is the gap between comb fingers, $h$ is thickness of the structures and $V$ is the applied voltage. It can be seen that the driving force is independent of the displacement. Therefore, the undesired "pull in" effect is avoided and a high tuning ratio can be realized.

The parallel plates, which have larger capacitance per unit area, are utilized as the capacitor in the structure. The capacitance as a function of the distance between parallel plates is described as

$$
C=\frac{n_{1} \varepsilon_{0} A}{d_{1}-\Delta d_{1}}
$$

where $n_{l}$ is the number of pairs of parallel plates, $d_{l}$ and $\Delta d_{l}$ are the distance and distance change between the plates, $A$ is the area 
of the plates. Compared to the comb-finger capacitors (where the overlap is changed), the parallel plate element requires a smaller displacement to achieve the same tuning ratio, and therefore requires a lower actuation voltage. As shown in the outlined box in Fig. 1, the movable plate (finger) in a parallel plate capacitor cell and the fixed plate in the adjacent cell form a parasitic capacitor with distance $d_{2}$. When $d_{1}$ decreases under the applied voltage, $d_{2}$ will increase. Consequently, the capacitance and capacitance change will deviate from equation (2). Similarly, a parasitic capacitor is also formed in the actuation section, which will decrease the actuation force. The effect is considered in our models, however it will not be discussed in detail here. To minimize the effect, $d_{2}$ is designed to be much larger than $d_{l}$.

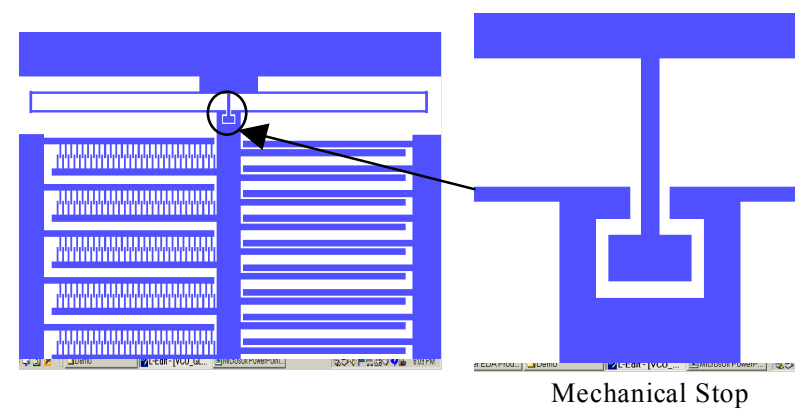

Fig. 2 A layout of our capacitor

Fig. 2 shows a layout of our capacitor. Though the gap between capacitor plates can be precisely controlled by comb drives, the plates may still touch due to mechanical shock or a voltage overshoot. A mechanical stop was designed to protect the parallel plates from shorting or failing because of stiction. The mechanical stop limits the minimum gap between the plates to be $0.5 \mu \mathrm{m}$ in our design, but it can be made smaller. The maximum tuning ratios for the capacitors with the initial gaps of $2 \mu \mathrm{m}, 3 \mu \mathrm{m}, 4 \mu \mathrm{m}$ and $5 \mu \mathrm{m}$ are $4: 1,6: 1,8: 1$ and $10: 1$, respectively, though there is no theoretical limitation if the mechanical stop is not present.

For comparison, we also designed a variable capacitor with parallelplate actuators and parallel-plate capacitors (shown in Fig. 3, and designated the Type I capacitor), and a variable capacitor with a comb-drive actuator and comb-finger capacitors (shown in Fig. 4, and designated the Type II capacitor).

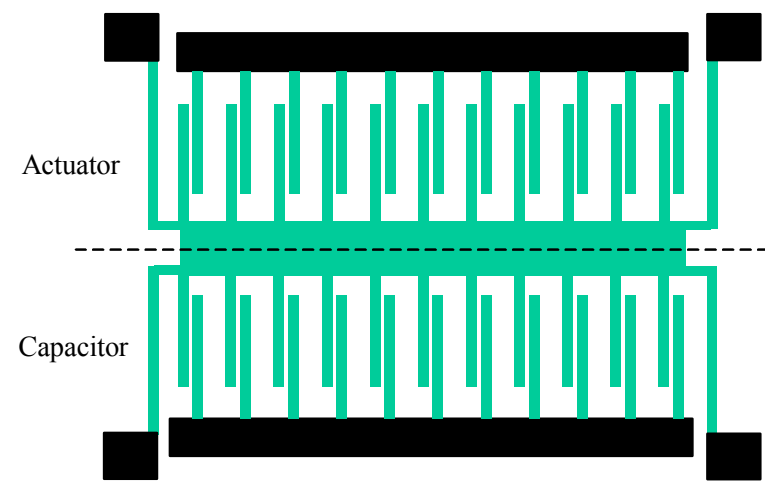

Fig.3 The schematic view of a gap-tuning capacitor with parallel plate actuator and parallel plate capacitor (Type I).

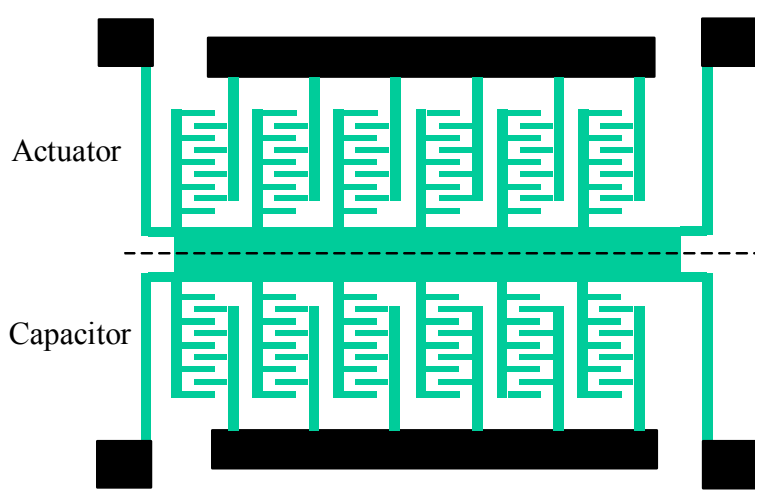

Fig.4 The schematic view of an area-tuning capacitor with comb-drives actuator and comb-finger capacitor (Type II)

\section{FABRICATION}

The capacitor is fabricated in a single layer of bulk micromachined single-crystal silicon that sits on a glass wafer. This simple process flow combines wafer bonding and DRIE (Deep Reactive Ion Etch) technologies (shown in Fig. 5). The process started with (100) heavily n-doped 4" silicon wafers. Shallow trenches approximately $4 \mu \mathrm{m}$ in depth were either dry etched by DRIE or wet etched with $\mathrm{KOH}$ (Fig. 5(a)). The silicon and glass wafers were anodically bonded in a $1000 \mathrm{~V}, 400^{\circ} \mathrm{C}$ and 1atm. ambient (Fig. 5(b)), in a Karl Suss wafer bonder. Silicon wafers were thinned to the desired thickness by wet etching with $\mathrm{KOH}$ (Fig. 5(c)). The thickness depends on structure specifications and process capability (about $50 \mu \mathrm{m}$ in our experiments). Finally, the structures were released by DRIE, and an aluminum layer was sputtered on the silicon structures to decrease series resistance and increase Q (Fig. 5(d)).

Compared to surface micromachining, bulk micromachining technology allows the creation of high-aspect-ratio singlecrystalline-silicon structures, enabling the fabrication of devices with high electrical and mechanical performance. Employing a glass substrate instead of a silicon one, will significantly reduce the parasitic capacitances due to the substrate for the variable capacitors.
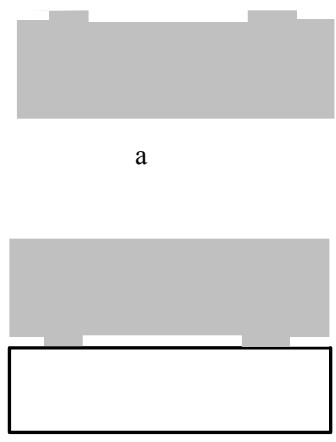

b

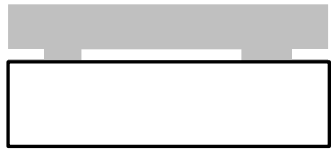

$\mathrm{c}$

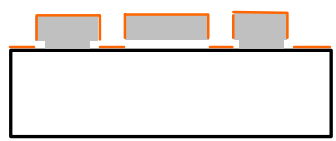

d

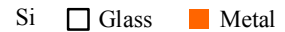

Fig. 5 The fabrication process of the variable capacitor. (a) etching a shallow trench, (b) anodic boding with Pyrex glass, (c) Thinning the silicon wafer, and (d) DRIE and sputtering metal. 


\section{RESULTS AND DISCUSSION}

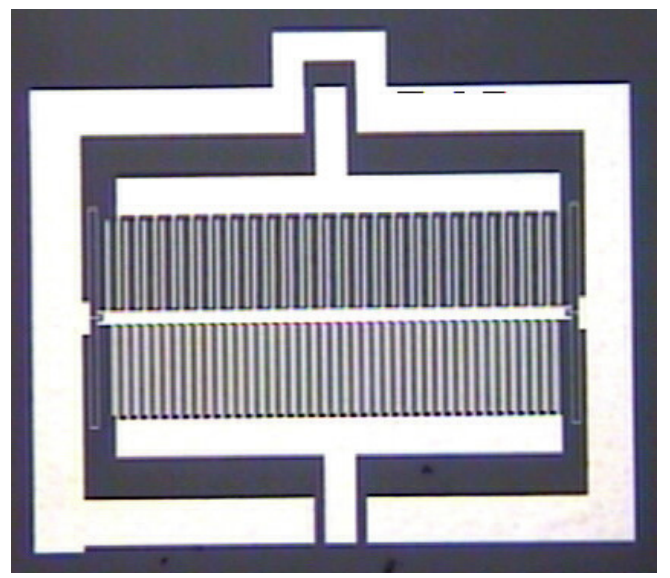

(a)

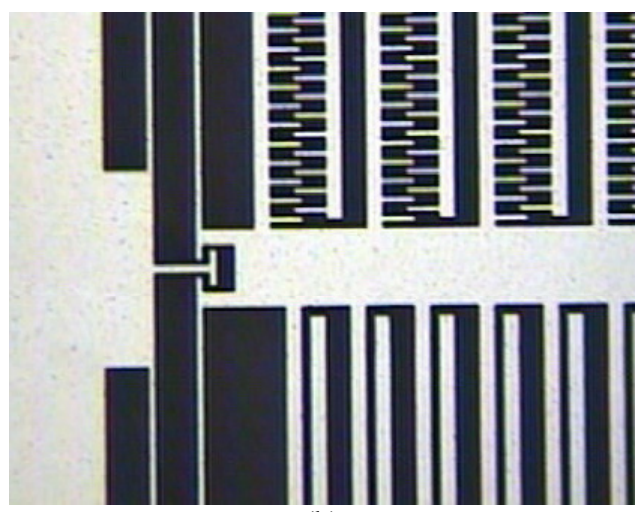

(b)

Fig.6 Micrographs of our variable capacitor: (a) entire device, and (b) a close-up view.

Fig. 6 shows micrographs of a fabricated variable capacitor. The space between the comb fingers is $3 \mu \mathrm{m}$. The width of the comb fingers and springs are also $3 \mu \mathrm{m}$. The gaps between the parallel plates, $d_{1}$, are $4 \mu \mathrm{m}$ and $5 \mu \mathrm{m}$ in different designs, and $d_{2}$ is $15 \mu \mathrm{m}$, at least 3 times $d_{l}$.

Fig. 7 presents the calculated and measured capacitance as a function of actuation voltage for our capacitor with different gap distances between plates $(2,3,4$ and $5 \mu \mathrm{m}$, respectively.) In our experiments, only capacitors with gaps of $4 \mu \mathrm{m}$ and $5 \mu \mathrm{m}$ were initially fabricated. The values of the capacitors with gaps of $4 \mu \mathrm{m}$ and $5 \mu \mathrm{m}$ are $2.0 \mathrm{pF}$ and $1.67 \mathrm{pF}$ at $0 \mathrm{~V}$, respectively. According to the discussion in the previous section, the designed tuning ratios are limited from $4: 1$ to $10: 1$ by a mechanical stop. However, the calculated tuning ratios are slightly less than those values, because of the parasitic capacitors that were included in our calculations with MATLAB. The measured maximum tuning ratio for the capacitor with a starting gap of $5 \mu \mathrm{m}$ is about $5: 1$, with a capacitance of $8.46 \mathrm{pF}$ at $20 \mathrm{~V}$. For the capacitor with a starting gap of $4 \mu \mathrm{m}$, the measured maximum capacitance is $8.23 \mathrm{pF}$ at $18 \mathrm{~V}$. These tuning ratios area smaller than designed, probably due to the gap of the fabricated mechanical stop being smaller than the design values because of lithography and etch bias. It was observed that the minimum space between the plates is approximately $1 \mu \mathrm{m}$ instead of $0.5 \mu \mathrm{m}$.

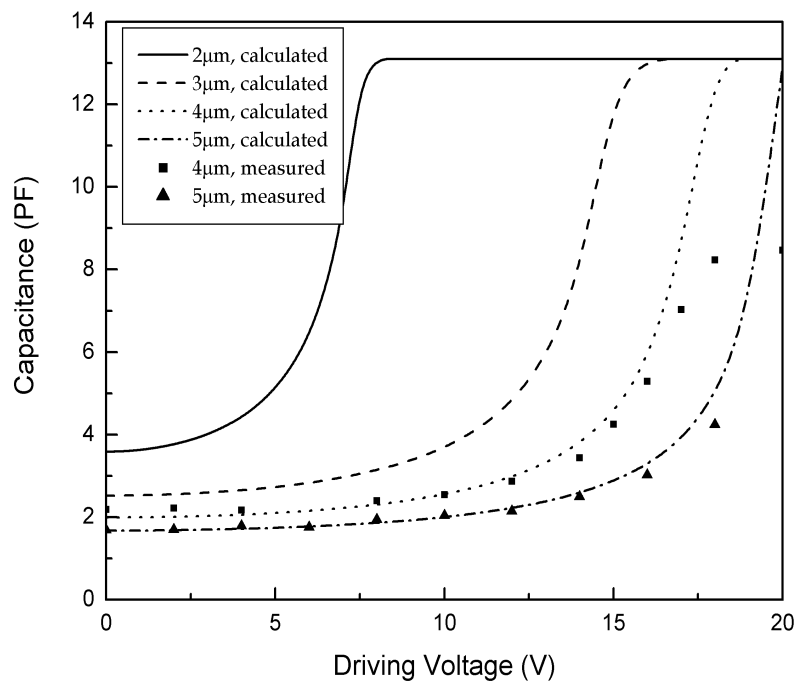

Fig. 7 The calculated and measured capacitances of our devices with different gaps between plates. For the capacitor with $2 \mu \mathrm{m}$ gap, the width and space of comb-drives are also $2 \mu \mathrm{m}$, while others are $3 \mu \mathrm{m}$.

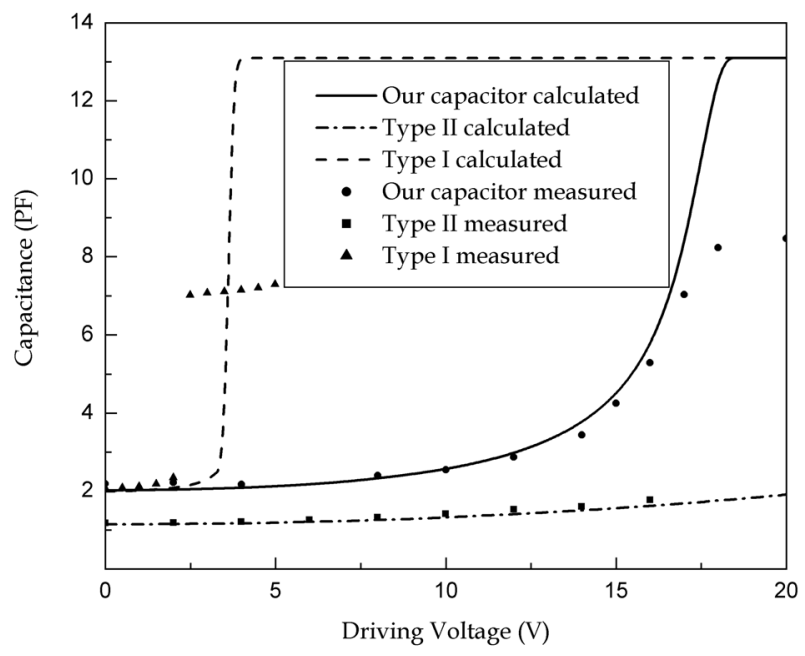

Fig.8 The comparison of calculated and measured capacitances for our capacitor, Type I and Type II capacitors. The gap between parallel plates is $4 \mu \mathrm{m}$, and the width and space of comb-drives are $3 \mu \mathrm{m}$.

Fig. 8 shows a comparison of our capacitor with Type I and Type II capacitors, where the gap between parallel plates is $4 \mu \mathrm{m}$ and the starting overlap of comb fingers is $5 \mu \mathrm{m}$. Our capacitor has larger capacitances at $0 \mathrm{~V}, 2.0 \mathrm{pF}$, compared to the Type II capacitor, which had a capacitance of $1.14 \mathrm{pF}$. The maximum measured capacitance of our capacitor is $8.23 \mathrm{pF}$ with tuning ratio of $4.1: 1$ at $18 \mathrm{~V}$, while the capacitance of the Type II capacitor at $18 \mathrm{~V}$ is only $1.8 \mathrm{pF}$ with tuning ratio of $1.6: 1$. It can be seen that our capacitor has higher initial capacitance and requires significantly lower driving voltages to achieve same tuning ratio 
when compared to the Type II capacitor. The Type I capacitor has the same capacitance as our capacitor at $0 \mathrm{~V}$, but the maximum measured capacitance is only $2.4 \mathrm{PF}$ with a tuning ratio of 1.2 , after which the plates are pulled in and snap together. The maximum measured tuning ratio is even lower than the theoretical value, 1.5:1, because of mechanical noise or electrical overshoot. The results show that our capacitor has much higher tuning range than the Type I capacitor.

Table. 1 compares the requirements of our capacitor and a Type II capacitor for given specifications of $2.5 \mathrm{pF}$ initial capacitance and $5: 1$ tuning range at $15 \mathrm{~V}$. It can be seen that the device area of our capacitor is less than half that of the Type II capacitor. Therefore, our capacitor allows for higher level of integration. The spring stiffness of our capacitor, $7.44 \mathrm{~N} / \mathrm{m}$, is much higher than that of the Type II capacitor, which was $1.74 \mathrm{~N} / \mathrm{m}$. As a result, our capacitor has lower mechanical noise sensitivity. In addition, shorter and/or wider springs will decrease series resistance and parasitic inductance, resulting in a higher $\mathrm{Q}$ as well as a higher self-resonant frequency. The resonant frequency of our capacitor, $2.17 \mathrm{kHz}$, is three times that of the Type II capacitor, which was $0.707 \mathrm{kHz}$. Therefore, our capacitor is capable of operating higher tuning speeds. We did not calculate the performance for the Type I capacitor, since it could not meet the tuning ratio the requirements.

\section{CONCLUSION}

We developed a variable capacitor in which the actuation is provided by comb drives and parallel plates are used for the capacitors. This capacitor has both the advantages of parallel-plate capacitors and the benefits found with comb-drive actuation. The comb drives allow for a high tuning ratio without the undesired "pull in" effect present with parallel plate actuators. In addition, the parallel plates provide a large capacitance per unit area and enable lower driving voltages than comb finger capacitors because a shorter displacement is required for an equivalent tuning ratio. This device requires a smaller area, has stiffer springs and a higher resonant frequency, which result in denser integration, low series resistance, low parasitic inductance, low mechanical noise sensitivity and high tuning speed.

\section{ACKNOWLEDGEMENT}

The authors would like to thank Prof. Kan of Cornell University who provided test instrumentation, Dr. Zengtao
Liu of Cornell University for help in measurement and Mr. Xiaosong Tang for assistance in the fabrication. Fabrication was done in the Cornell Nanofabrication Facility.

\section{REFERENCE}

1. C. T.-C. Nguyen, L. P. B. Katehi and G. M. Rebeiz, "Micromachined Devices For Wireless Communications," Proc. IEEE, vol. 86, no. 8, 1998, pp. 1756-68

2. J. J. Yao, "RF MEMS from a device perspective," J. Micromech. Microeng. 10 (2000), R9-R38

3. J. J. Yao, S. T. Park, R. Anderson and J. DeNatale, "High Tuning Ratio MEMS-Based Tunable Capacitors For RF Communications", Proc. Solid-state Sensor and Actuator Workshop, Hilton Head Island, 1998

4. A. Dec and K. Suyama, "Micromachined Electro-Mechanically Tunable Capacitors And Their Applications For RF IC's" IEEE J. Solid-State Circuits, vol. 35, no. 8, 2000, pp. 1231-37

5. J. Zou, C. Liu, J. S-. Aline, J. Chen, S.-Mo Kang, "Development Of A Wide Tuning Range MEMS Tunable Capacitor For Wireless Communication Systems", IEDM 2000, 2000, p403-406.

6. D. J. Young and B. E. Boser, "A Micromachined Variable Capacitor For Monolithic Low-Noise VCOs," in Proc. IEEE Solid-State Sensors Actuators Workshop, Hilton Head, SC, 1996, pp. 86-89

7. D. J. Young, J. L. Tham, and B. E. Boser, A MicromachineBased Low Phase-Noise GHz Voltage-Controlled Oscillator for Wireless Communications, The 10th International Conference on Solid- State Sensors and Actuators (Transducers'99), Sendai, Japan, 1999, P1386

8. J. J. Yao, S. T. Park, and J. DeNatale, "A Low Power/Low Voltage Electrostatic Actuator For RF MEMS Applications," Proc. Solid-state Sensor and Actuator Workshop, Hilton Head Island, 2000, pp. 246-49

9. A. L. Pisano, and Y. H. Cho, " Mechanical Design Issues in Laterally-driven Microstructures", Sensors and Actuators, A21-23, 1990, 1060-1064

10. W. C. Tang, T.-C. H. Nguyen, M. W. Judy, and R. T. Howe, "Electrostatic-Comb Drive Of Lateral Polysilicon Resonators," Sensors Actuators, vol. A21, pp. 328-331, Feb. 1990.

Table.1. The device requirements for our capacitor and the Type II capacitor given equivalent specifications. The initial overlap of the comb fingers is $5 \mu \mathrm{m}$.

\begin{tabular}{|l|l|c|l|l||l|l|l|}
\hline \multirow{2}{*}{ Devices } & \multicolumn{4}{|c|}{ Specifications } & \multicolumn{3}{|c|}{ Requirements } \\
\cline { 2 - 8 } & $\begin{array}{l}\text { Feature } \\
\text { Size }\end{array}$ & Initial Capacitance & $\begin{array}{l}\text { Tuning } \\
\text { Ratio }\end{array}$ & $\begin{array}{l}\text { Driving } \\
\text { Voltage }\end{array}$ & $\begin{array}{l}\text { Device } \\
\text { Area }\end{array}$ & $\begin{array}{l}\text { Spring } \\
\text { Stiffness }\end{array}$ & $\begin{array}{l}\text { Resonant } \\
\text { Frequency }\end{array}$ \\
\hline Ours & $3 \mu \mathrm{m}$ & $2.5 \mathrm{pF}$ & $5: 1$ & $15 \mathrm{~V}$ & $1.5 \mathrm{~mm}^{2}$ & $7.44 \mathrm{~N} / \mathrm{m}$ & $2.17 \mathrm{kHz}$ \\
\hline Type II & $3 \mu \mathrm{m}$ & $2.5 \mathrm{pF}$ & $5: 1$ & $15 \mathrm{~V}$ & $3.3 \mathrm{~mm}^{2}$ & $1.74 \mathrm{~N} / \mathrm{m}$ & $0.707 \mathrm{kHz}$ \\
\hline
\end{tabular}

